\title{
Gamma-convergence of Generalized Gradient Flows with Conjugate Type
}

\author{
Mao-Sheng Chang* and Jian-Tong Liao
}

\begin{abstract}
In this paper we establish the Gamma-convergence of generalized gradient flows with conjugate type. It provided a criteria for obtaining the convergence of generalized gradient flows that correspond to a sort of $C^{1}$-order $\Gamma$-convergence of energy functionals and a kind of bounded symmetric positive definite linear operators.
\end{abstract}

\section{Introduction}

$\Gamma$-convergence theory [2,8] was introduced by Ennio De Giorgi in 1970s. It provided a useful criteria to obtain the convergence of global minimizers of energy functional $E_{\epsilon}$ to global minimizers of a limiting functional $F$.

The notion of $\Gamma$-convergence of gradient flows was introduced by E. Sandier and S. Serfaty in 2004. It's a time-dependent notion of the $\Gamma$-convergence. It provided a useful criteria to obtain the convergence of gradient flows that correspond to a sort of $C^{1}$-order $\Gamma$-convergence of energy functionals [18]. This abstract method was used successfully for the dynamics of Ginzburg-Landau vortices [18, the Cahn-Hilliand equation [13, 14, and the Allen-Cahn equation [20]. A generalization of this notion to metric spaces was developed by Serfaty [20] and a discussion on a nonlinear system case is contained in [5]. This give us a strong motivation for the study of Gamma-convergence of generalized gradient flows (1.2) in the sense of Sandier and Serfaty [18].

Suppose that $X$ is a real Hilbert space with inner product $\langle\cdot, \cdot\rangle_{X}$ and corresponding norm $\|\cdot\|_{X}$. Let $E \in C^{1}(X ; \mathbb{R})$ and let $\gamma: I \subseteq \mathbb{R} \mapsto X$ be a differentiable curve in $X$ with $\gamma\left(t_{0}\right)=x \in X$. Then

$$
\begin{aligned}
\left.\frac{d}{d t}\right|_{t=t_{0}} E(\gamma(t)) & =\left\langle\nabla_{X} E\left(\gamma\left(t_{0}\right)\right), \gamma^{\prime}\left(t_{0}\right)\right\rangle_{X} \\
& =\left\|\nabla_{X} E\left(\gamma\left(t_{0}\right)\right)\right\|_{X} \cdot\left\|\gamma^{\prime}\left(t_{0}\right)\right\|_{X} \cdot \cos \theta,
\end{aligned}
$$

where $\theta$ is the angle between $\nabla_{X} E\left(\gamma\left(t_{0}\right)\right)$ and $\gamma^{\prime}\left(t_{0}\right)$ in the sense of geometry. Assume that $u: I \mapsto X$ is a solution of the gradient flow

$$
\partial_{t} u(t)=-\nabla_{X} E(u(t)) \in X, \quad \forall t \in I .
$$

Received August 22, 2021; Accepted November 21, 2021.

Communicated by Jenn-Nan Wang.

2020 Mathematics Subject Classification. Primary: 47J35, 49J40; Secondary: 49Q20.

Key words and phrases. Gamma-convergence of gradient flow, real Hilbert space, linear operator, fractional Laplacian operator, $\Gamma$-convergence, fractional gradient flow.

*Corresponding author. 
Obviously, the direction of $\nabla_{X} E(u(t))$ and $\partial_{t} u(t)$ is opposite. Hence, the curve (solution) $u$ of $(1.1)$ is the best path for decreasing the energy $E$ with steepest descent. Evolution equations with the corresponding gradient flow have been of special interest in analysis and mathematical physics since 2001. Many problems in computer vision have been formulated as gradient flows of various energies use an inner product to define the notion of gradient. The idea of "optimization by gradient flows" is particularly useful in tracking applications. In most application cases in computer vision, a common minimization procedure consists in evolving an initial contour, positioned by the user, in the direction of steepest descent of the energy. This approach, known in the literature as active contours or deformable models, was pioneered by Kass, Witkin, and Terzopoulos in [10 for the purpose of image segmentation.

We are interested in the following generalized gradient flows:

$$
\partial_{t} u=-L\left(\nabla_{X} E(u)\right)
$$

where $\nabla_{X} E(u)$ is the gradient of $E$ relative to the inner product $\langle\cdot, \cdot\rangle_{X}$ at $u$, and $L: X \mapsto$ $X$ is an invertible, symmetric, positive definite, and linear operator. Charpiat, Maurel, Pons, Keriven, and Faugeras 6 devoted to the general gradient descent process and a rewriting of the usual definition of the gradient shows how the choice of an inner product can be seen as a way to introduce a priori on the deformation fields and forms a natural extension of the notion of gradient to more general priors.

Following the pioneering work by Otto [16], many PDE problems have been translated into gradient flow problems [1] and gradient flows has become a great strategy for the study of dissipation evolution problems.

In this paper we establish the Gamma-convergence of generalized gradient flows with conjugate type. It provided a criteria for obtaining the convergence of generalized gradient flows that correspond to a sort of $C^{1}$-order $\Gamma$-convergence of energy functionals and a kind of bounded symmetric positive definite linear operators. The paper is organized as follows. In Section 1, the background and main goal of our paper are introduced. Motivations and related preliminary tools will be recalled in Section 2. Section 3 is devoted to the main theorems and its proofs.

\section{Motivations and some elementary results}

Let $\left(X,\langle\cdot, \cdot\rangle_{X}\right)$ be a real Hilbert space (hence $\left(X,\|\cdot\|_{X}\right)$ is a Banach space with norm $\left.\|u\|_{X} \equiv \sqrt{\langle u, u\rangle_{X}}\right)$. Let $E: X \mapsto \mathbb{R}$ be a functional defined on $X$. We say that $E$ is Fréchet differentiable at $u(\in X)$ if there exists a (unique) bounded linear functional $D E(u): X \mapsto \mathbb{R}$ (i.e., $D E(u) \in X^{*} \equiv \mathcal{L}(X ; \mathbb{R})=$ the space of all bounded linear functionals 
on $X ; X^{*}$ is called the dual space of $X$.) such that

$$
\lim _{\|h\|_{X \mapsto 0}} \frac{E(u+h)-E(u)-D E(u)(h)}{\|h\|_{X}}=0 .
$$

Suppose that $E$ is Fréchet differentiable at $u$. Using the Riesz Representation theorem, there exists a unique element $\widehat{u} \in X$ such that

$$
D E(u)(v)\left(=\langle D E(u), v\rangle_{X^{*}, X}\right)=\langle\widehat{u}, v\rangle_{X}, \quad \forall v \in X .
$$

Let we write

$$
\nabla_{X} E(u) \equiv \widehat{u}
$$

$\nabla_{X} E(u)$ is called the gradient of $E$ at $u$. (Note that $\nabla_{X} E(u) \in X$.)

Let $u \in X$ and $v \in X$ with $v \neq 0$. The first variation of $E$ at $u$ in the direction $v$, $\delta E(u, v)$, is defined by

$$
\delta E(u, v) \equiv \lim _{t \rightarrow 0} \frac{E(u+t v)-E(u)}{t}=\left.\frac{d}{d t}(E(u+t v))\right|_{t=0} .
$$

Assume that $E$ is Fréchet differentiable at $u$. Then $\delta E(u, v)$ exists for each $v \in X$ with $v \neq 0$, and

$$
\delta E(u, v)=D E(u)(v)=\left\langle\nabla_{X} E(u), v\right\rangle_{X} \quad \text { for all } v \in X \text { with } v \neq 0 .
$$

Let $\gamma: \mathbb{R} \mapsto X$ be a differentiable curve in $X$. Then

$$
\left.\frac{d}{d t}(E(\gamma(t)))\right|_{t=\tau}=\left\langle\nabla_{X} E(\gamma(\tau)), \gamma^{\prime}(\tau)\right\rangle_{X}, \quad \forall \tau \in \mathbb{R}
$$

Moreover, for any $t_{0}, t_{1} \in \mathbb{R}$, we have

$$
E\left(\gamma\left(t_{1}\right)\right)-E\left(\gamma\left(t_{0}\right)\right)=\int_{t_{0}}^{t_{1}} \frac{d}{d t}(E(\gamma(t))) d t=\int_{t_{0}}^{t_{1}}\left\langle\nabla_{X} E(\gamma(t)), \gamma^{\prime}(t)\right\rangle_{X} d t
$$

Suppose that $\gamma:[0, T] \mapsto X$ is a solution of the gradient flow

$$
\gamma^{\prime}(t)=-\nabla_{X} E(\gamma(t)), \quad \forall t \in[0, T]
$$

Then we have

$$
\begin{aligned}
E(\gamma(t))-E(\gamma(0)) & =\int_{0}^{t} \frac{d}{d t}(E(\gamma(\tau))) d \tau=\int_{0}^{t}\left\langle\nabla_{X} E(\gamma(\tau)),-\nabla_{X} E(\gamma(\tau))\right\rangle_{X} d \tau \\
& =-\int_{0}^{t}\left\|\nabla_{X} E(\gamma(\tau))\right\|_{X}^{2} d \tau=-\int_{0}^{t}\left\|\gamma^{\prime}(\tau)\right\|_{X}^{2} d \tau \leq 0, \quad \forall t \in[0, T]
\end{aligned}
$$

Suppose that $f(x, u, p): \mathbb{R}^{n} \times \mathbb{R}^{1} \times \mathbb{R}^{n} \mapsto \mathbb{R}^{1}$ is a smooth function. Assume that $\Omega$ is a bounded domain in $\mathbb{R}^{n}$ with smooth boundary. Let $E: L^{2}(\Omega) \mapsto \mathbb{R}$ be defined by

$$
E(u) \equiv \int_{\Omega} f\left(x, u(x), \frac{\partial u}{\partial x_{1}}(x), \ldots, \frac{\partial u}{\partial x_{n}}(x)\right) d x .
$$


For the given function $u: \Omega \mapsto \mathbb{R}$, we define $h^{u}: \Omega \mapsto \mathbb{R}^{n} \times \mathbb{R}^{1} \times \mathbb{R}^{n}$ by

$$
h^{u}(x) \equiv(x, u(x), \nabla u(x)) \quad \text { for all } x=\left(x_{1}, \ldots, x_{n}\right) \in \Omega
$$

Then

$$
\begin{aligned}
E(u) & =\int_{\Omega}\left(f \circ h^{u}\right)(x) d x \\
\delta E(u, v) & =\int_{\Omega}\left(f_{u} \circ h^{u}-\sum_{i=1}^{n} \frac{\partial}{\partial x_{i}}\left(f_{p_{i}} \circ h^{u}\right)\right)(x) \cdot v(x) d x \\
& =\left\langle f_{u} \circ h^{u}-\sum_{i=1}^{n} \frac{\partial}{\partial x_{i}}\left(f_{p_{i}} \circ h^{u}\right), v\right\rangle_{L^{2}(\Omega)} \quad \text { for all } v \in L^{2}(\Omega) .
\end{aligned}
$$

Here, $f_{u} \equiv \frac{\partial f}{\partial u}, f_{p_{i}} \equiv \frac{\partial f}{\partial p_{i}}\left(x_{1}, \ldots, x_{n}, u, p_{1}, \ldots, p_{n}\right)$. Therefore we have

$$
\nabla_{L^{2}(\Omega)} E(u)=f_{u} \circ h^{u}-\sum_{i=1}^{n} \frac{\partial}{\partial x_{i}}\left(f_{p_{i}} \circ h^{u}\right) \in L^{2}(\Omega) .
$$

If $\delta E(u, v)=0$ for all $v \in L^{2}(\Omega)$, then $u$ satisfies the Euler-Lagrange equation

$$
\sum_{i=1}^{n} \frac{\partial}{\partial x_{i}}\left(f_{p_{i}} \circ h^{u}\right)(x)-\left(f_{u} \circ h^{u}\right)(x)=0 \quad \text { for a.e. } x \in \Omega .
$$

Let $\mathcal{S}$ be the Schwartz space of rapidly decaying $C^{\infty}\left(\mathbb{R}^{n}\right)$ functions. The fractional Laplacian of $u \in \mathcal{S},(-\Delta)^{s} u$, is defined by

$$
(-\Delta)^{s} u \equiv \mathcal{F}^{-1}\left(|\xi|^{2 s}(\mathcal{F} u)(\xi)\right)
$$

where

$$
(\mathcal{F} u)(\xi) \equiv \frac{1}{(2 \pi)^{n / 2}} \int_{\mathbb{R}^{n}} e^{-i \xi \cdot x} u(x) d x, \quad \xi \in \mathbb{R}^{n}
$$

denotes the Fourier transform of $u$. See 9, 21 for the basic properties of the operator $(-\Delta)^{s}$. Let $H^{s}\left(\mathbb{R}^{n}\right)$ denote the fractional Sobolev space of the functions $u$ in $L^{2}\left(\mathbb{R}^{n}\right)$ such that

$$
\text { the function }(x, y) \mapsto \frac{u(x)-u(y)}{|x-y|^{n / 2+s}} \text { is in } L^{2}\left(\mathbb{R}^{n} \times \mathbb{R}^{n}, d x d y\right) \text {. }
$$

The nonlocal operator $(-\Delta)^{s}: \mathcal{S} \rightarrow L^{2}\left(\mathbb{R}^{n}\right)$ can also be expressed by

$$
\begin{aligned}
(-\Delta)^{s} u(x) & \equiv C(n, s) \lim _{\epsilon \rightarrow 0^{+}} \int_{\mathbb{R}^{n} \backslash B(x, \epsilon)} \frac{u(x)-u(y)}{|x-y|^{n+2 s}} d y \\
& =C(n, s) \mathrm{P} . \mathrm{V} \cdot \int_{\mathbb{R}^{n}} \frac{u(x)-u(y)}{|x-y|^{n+2 s}} d y
\end{aligned}
$$


where $s \in(0,1), B(x, \epsilon)$ is the open ball centered at $x \in \mathbb{R}^{n}$ and radius $\epsilon$, and $C(n, s)$ is the positive normalization constant

$$
C(n, s) \equiv\left(\int_{\mathbb{R}^{n}} \frac{1-\cos \left(\zeta_{1}\right)}{|\zeta|^{n+2 s}} d \zeta\right)^{-1}
$$

Recalling the following formulas which is proved in 9,17 :

(i) $(-\Delta)^{s} u=\mathcal{F}^{-1}\left(|\xi|^{2 s}(\mathcal{F} u)(\xi)\right)$,

(ii) $[u]_{H^{s}\left(\mathbb{R}^{n}\right)}^{2} \equiv \int_{\mathbb{R}^{n} \times \mathbb{R}^{n}} \frac{|u(x)-u(y)|^{2}}{|x-y|^{n+2 s}} d x d y=\frac{2}{C(n, s)} \int_{\mathbb{R}^{n}}|\xi|^{2 s}|\mathcal{F} u(\xi)|^{2} d \xi$,

(iii) $[u]_{H^{s}\left(\mathbb{R}^{n}\right)}^{2}=\frac{2}{C(n, s)}\left\|(-\Delta)^{s / 2} u\right\|_{L^{2}\left(\mathbb{R}^{n}\right)}^{2}$,

(iv) (Sobolev trace equality)

$$
\left\|(-\Delta)^{s / 2} u\right\|_{L^{2}\left(\mathbb{R}^{n}\right)}^{2}=\int_{\mathbb{R}^{n}}\left((-\Delta)^{s} u(x)\right) u(x) d x=\int_{\mathbb{R}^{n}}|\xi|^{2 s}|\mathcal{F} u(\xi)|^{2} d \xi
$$

for each $u \in H^{s}\left(\mathbb{R}^{n}\right)$. Let we define the Hilbert space $\widehat{H}^{s}\left(\mathbb{R}^{n}\right)$ by means of the Fourier transform as follows:

$$
\widehat{H}^{s}\left(\mathbb{R}^{n}\right) \equiv\left\{\left.u \in L^{2}\left(\mathbb{R}^{n}\right)\left|\int_{\mathbb{R}^{n}}\left(1+|\xi|^{2 s}\right)\right| \mathcal{F} u(\xi)\right|^{2} d \xi<+\infty\right\} .
$$

In this case, the inner product and the norm are given by

$$
\begin{aligned}
\langle u, v\rangle_{\widehat{H}^{s}\left(\mathbb{R}^{n}\right)} & \equiv \int_{\mathbb{R}^{n}}\left(1+|\xi|^{2 s}\right) \mathcal{F} u(\xi) \mathcal{F} v(\xi) d \xi \\
\|u\|_{\widehat{H}^{s}\left(\mathbb{R}^{n}\right)} & \equiv \sqrt{\langle u, u\rangle_{\widehat{H}^{s}\left(\mathbb{R}^{n}\right)}}=\left(\int_{\mathbb{R}^{n}}\left(1+|\xi|^{2 s}\right)|\mathcal{F} u(\xi)|^{2} d \xi\right)^{1 / 2} .
\end{aligned}
$$

Let we define another inner product $\langle\cdot, \cdot\rangle_{H^{s}\left(\mathbb{R}^{n}\right)}$ on $H^{s}\left(\mathbb{R}^{n}\right)$ by

$$
\langle u, v\rangle_{H^{s}\left(\mathbb{R}^{n}\right)} \equiv\langle u, v\rangle_{L^{2}\left(\mathbb{R}^{n}\right)}+\left\langle(-\Delta)^{s / 2} u,(-\Delta)^{s / 2} v\right\rangle_{L^{2}\left(\mathbb{R}^{n}\right)} .
$$

The norm $\|\cdot\|_{H^{s}\left(\mathbb{R}^{n}\right)}$ of this Hilbert space $H^{s}\left(\mathbb{R}^{n}\right)$ is given by

$$
\begin{aligned}
\|u\|_{H^{s}\left(\mathbb{R}^{n}\right)}^{2} & =\|u\|_{L^{2}\left(\mathbb{R}^{n}\right)}^{2}+\left\|(-\Delta)^{s / 2} u\right\|_{L^{2}\left(\mathbb{R}^{n}\right)}^{2} \\
& =\|u\|_{L^{2}\left(\mathbb{R}^{n}\right)}^{2}+\frac{C(n, s)}{2}[u]_{H^{s}\left(\mathbb{R}^{n}\right)}^{2} \\
& =\|u\|_{L^{2}\left(\mathbb{R}^{n}\right)}^{2}+\int_{\mathbb{R}^{n}}|\xi|^{2 s}|\mathcal{F} u(\xi)|^{2} d \xi
\end{aligned}
$$

Note that

$$
\begin{aligned}
\left\langle(-\Delta)^{s / 2} u,(-\Delta)^{s / 2} v\right\rangle_{L^{2}\left(\mathbb{R}^{n}\right)} & =\int_{\mathbb{R}^{n}}(-\Delta)^{s / 2} u(x)(-\Delta)^{s / 2} v(x) d x \\
& =\frac{C(n, s)}{2} \int_{\mathbb{R}^{n}} \int_{\mathbb{R}^{n}} \frac{(u(x)-u(y))(v(x)-v(y))}{|x-y|^{n+2 s}} d x d y .
\end{aligned}
$$


Using the classical Plancherel's theorem, we have

$$
\|u\|_{L^{2}\left(\mathbb{R}^{n}\right)}^{2}=\|\mathcal{F} u\|_{L^{2}\left(\mathbb{R}^{n}\right)}^{2}=\int_{\mathbb{R}^{n}}|(\mathcal{F} u)(\xi)|^{2} d \xi
$$

Suppose that the inner product $(\cdot, \cdot)_{H^{s}\left(\mathbb{R}^{n}\right)}$ on $H^{s}\left(\mathbb{R}^{n}\right)$ is defined by

$$
(u, v)_{H^{s}\left(\mathbb{R}^{n}\right)} \equiv\langle u, v\rangle_{L^{2}\left(\mathbb{R}^{n}\right)}+\frac{C(n, s)}{2} \int_{\mathbb{R}^{n}} \int_{\mathbb{R}^{n}} \frac{(u(x)-u(y))(v(x)-v(y))}{|x-y|^{n+2 s}} d x d y,
$$

and the norm $\|\cdot\|_{H^{s}\left(\mathbb{R}^{n}\right)}$ (induced by $\left.(\cdot, \cdot)_{H^{s}\left(\mathbb{R}^{n}\right)}\right)$ is

$$
\|u\|_{H^{s}\left(\mathbb{R}^{n}\right)}^{2}=\|u\|_{L^{2}\left(\mathbb{R}^{n}\right)}^{2}+\frac{C(n, s)}{2} \int_{\mathbb{R}^{n}} \int_{\mathbb{R}^{n}} \frac{|u(x)-u(y)|^{2}}{|x-y|^{n+2 s}} d x d y .
$$

Then the Hilbert space $H^{s}\left(\mathbb{R}^{n}\right)$ coincides with $\widehat{H}^{s}\left(\mathbb{R}^{n}\right)$ and the norms

$$
\begin{aligned}
\|u\|_{\widehat{H}^{s}\left(\mathbb{R}^{n}\right)} & \equiv\left(\int_{\mathbb{R}^{n}}\left(1+|\xi|^{2 s}\right)|\mathcal{F} u(\xi)|^{2} d \xi\right)^{1 / 2} \\
\|u\|_{H^{s}\left(\mathbb{R}^{n}\right)} & \equiv\left(\|u\|_{L^{2}\left(\mathbb{R}^{n}\right)}^{2}+\left\|(-\Delta)^{s / 2} u\right\|_{L^{2}\left(\mathbb{R}^{n}\right)}^{2}\right)^{1 / 2} \\
\|u\|_{H^{s}\left(\mathbb{R}^{n}\right)}^{*} & \equiv\left(\|u\|_{L^{2}\left(\mathbb{R}^{n}\right)}^{2}+\frac{C(n, s)}{2} \int_{\mathbb{R}^{n}} \int_{\mathbb{R}^{n}} \frac{|u(x)-u(y)|^{2}}{|x-y|^{n+2 s}} d x d y\right)^{1 / 2}
\end{aligned}
$$

and

$$
\|u\|_{H^{s}\left(\mathbb{R}^{n}\right)}^{* *} \equiv\left(\int_{\mathbb{R}^{n}}|u(x)|^{2} d x+\int_{\mathbb{R}^{n}}|\xi|^{2 s}|\mathcal{F} u(\xi)|^{2} d \xi\right)^{1 / 2}
$$

are equivalent.

Suppose that

$$
E_{\epsilon}(u)=\int_{\Omega} \frac{\epsilon \cdot|\nabla u|^{2}}{2}+\frac{1}{\epsilon} W(u)
$$

is the energy functional arising in the van der Waals-Cahn-Hilliard theory of phase transitions. Here $\Omega$ is a bounded smooth domain in $\mathbb{R}^{n}(n \geq 2), f(u)=W^{\prime}(u)$, and $W(u)=\frac{1}{2}\left(1-u^{2}\right)^{2}$. Let $(\cdot, \cdot)$ denote the pairing between $\left(H^{1}(\Omega)\right)^{*}$ and $H^{1}(\Omega)$. Define

$$
\begin{array}{r}
H^{-1}(\Omega)=\left\{f \in\left(H^{1}(\Omega)\right)^{*} \mid\right. \\
\text { there exists a unique }\left((-\Delta)^{-1} f\right) \in H^{1}(\Omega) \text { with } \\
\text { mean } 0 \text { over } \Omega \text { such that } \\
\left.(f, v)=\int_{\Omega} \nabla\left((-\Delta)^{-1} f\right) \cdot \nabla v \text { for all } v \in H^{1}(\Omega)\right\} .
\end{array}
$$

Observe that $H^{-1}(\Omega)$ is a real Hilbert space with inner product

$$
\langle u, v\rangle_{H^{-1}(\Omega)}=\int_{\Omega} \nabla\left((-\Delta)^{-1} u\right) \cdot \nabla\left((-\Delta)^{-1} v\right) d x \quad \text { for all } u, v \in H^{-1}(\Omega),
$$


and the corresponding norm

$$
\|u\|_{H^{-1}(\Omega)}=\left\|\nabla\left((-\Delta)^{-1} u\right)\right\|_{L^{2}(\Omega)}=\left(\int_{\Omega} \nabla\left((-\Delta)^{-1} u\right) \cdot \nabla\left((-\Delta)^{-1} u\right) d x\right)^{1 / 2} .
$$

Let $h^{u}: \Omega \mapsto \mathbb{R}^{n} \times \mathbb{R}^{1} \times \mathbb{R}^{n}$ be defined by

$$
h^{u}(x) \equiv(x, u(x), \nabla u(x)) \quad \text { for all } x \in \Omega,
$$

and let $f: \mathbb{R}^{n} \times \mathbb{R}^{1} \times \mathbb{R}^{n} \mapsto \mathbb{R}$ be defined by

$$
f(x, u, p) \equiv \frac{\epsilon|p|^{2}}{2}+\frac{1}{\epsilon} W(u), \quad(x, u, p) \in \mathbb{R}^{n} \times \mathbb{R}^{1} \times \mathbb{R}^{n} .
$$

Suppose that the functional $E_{\epsilon}$ is defined as

$$
E_{\epsilon}(u) \equiv \int_{\Omega}\left(f \circ h^{u}\right)(x) d x .
$$

Then

$$
\nabla_{L^{2}(\Omega)} E_{\epsilon}(u)=f_{u} \circ h^{u}-\sum_{i=1}^{n} \frac{\partial}{\partial x_{i}}\left(f_{p_{i}} \circ h^{u}\right) \in L^{2}(\Omega)
$$

where

$$
\begin{aligned}
f_{u}(x, u, p) & =\frac{\partial f}{\partial u}(x, u, p)=\frac{1}{\epsilon} W^{\prime}(u) \\
f_{p_{i}}(x, u, p) & =\frac{\partial f}{\partial p_{i}}\left(x, u, p_{1}, \ldots, p_{n}\right)=\epsilon p_{i}, \quad i=1,2, \ldots, n, \\
\left(f_{p_{i}} \circ h^{u}\right)(x) & =f_{p_{i}}(x, u(x), \nabla u(x))=\epsilon u_{x_{i}}(x)
\end{aligned}
$$

and

$$
\frac{\partial}{\partial x_{i}}\left(f_{p_{i}} \circ h^{u}\right)(x)=\frac{\partial}{\partial x_{i}}\left(\epsilon u_{x_{i}}\right)(x)=\epsilon u_{x_{i} x_{i}}(x)
$$

Thus,

$$
\begin{aligned}
\left(\nabla_{L^{2}(\Omega)} E_{\epsilon}(u)\right)(x) & =f_{u}(x, u(x), \nabla u(x))-\sum_{i=1}^{n} \epsilon u_{x_{i} x_{i}}(x) \\
& =\frac{1}{\epsilon} W^{\prime}(u(x))-\epsilon \Delta u(x)=\frac{1}{\epsilon}\left(W^{\prime} \circ u\right)(x)-\epsilon \Delta u(x) \\
& =\left(\frac{1}{\epsilon} W^{\prime} \circ u-\epsilon \Delta u\right)(x), \quad \forall x \in \Omega .
\end{aligned}
$$

Owing to

$$
\left.\frac{d}{d t}\left(E_{\epsilon}(u+t v)\right)\right|_{t=0}=\delta E_{\epsilon}(u, v)=\left\langle\nabla_{X} E_{\epsilon}(u), v\right\rangle_{X}, \quad \forall v \in X \text { with } v \neq 0,
$$


we have

$$
\begin{aligned}
\left.\frac{d}{d t}\left(E_{\epsilon}(u+t v)\right)\right|_{t=0} & =\left\langle\nabla_{L^{2}(\Omega)} E_{\epsilon}(u), v\right\rangle_{L^{2}(\Omega)}=\left\langle\nabla_{H^{-1}(\Omega)} E_{\epsilon}(u), v\right\rangle_{H^{-1}(\Omega)} \\
& =\left\langle\nabla_{H^{s}(\Omega)} E_{\epsilon}(u), v\right\rangle_{H^{s}(\Omega)}=\left\langle\nabla_{H^{1}(\Omega)} E_{\epsilon}(u), v\right\rangle_{H^{1}(\Omega)} .
\end{aligned}
$$

Since

$$
\begin{aligned}
\langle u, v\rangle_{H^{1}(\Omega)} & =\int_{\Omega} \sum_{|\alpha| \leq 1} D^{\alpha} u D^{\alpha} v d x=\int_{\Omega} u v d x+\int_{\Omega}\left(\sum_{i=1}^{n} u_{x_{i}} v_{x_{i}}\right) d x \\
& =\langle u, v\rangle_{L^{2}(\Omega)}+\int_{\Omega} \nabla u \cdot \nabla v d x=\langle u, v\rangle_{L^{2}(\Omega)}+\langle\nabla u, \nabla v\rangle_{L^{2}(\Omega)} \\
& =\langle u, v\rangle_{L^{2}(\Omega)}+\langle-\Delta u, v\rangle_{L^{2}(\Omega)}+\int_{\partial \Omega} v \frac{\partial u}{\partial \nu} d S \\
& =\langle(1+(-\Delta)) u, v\rangle_{L^{2}(\Omega)}+\int_{\partial \Omega} v \frac{\partial u}{\partial \nu} d S,
\end{aligned}
$$

under the suitable boundary conditions (for example, $v=0$ or $\frac{\partial u}{\partial \nu}=0$ on $\partial \Omega$ ), we have

$$
\langle u, v\rangle_{H^{1}(\Omega)}=\langle(1+(-\Delta)) u, v\rangle_{L^{2}(\Omega)} .
$$

The inner product of $u$ and $v$ in $H^{-1}(\Omega)$ is

$$
\begin{aligned}
\langle u, v\rangle_{H^{-1}(\Omega)} & =\left\langle\nabla\left((-\Delta)^{-1} u\right), \nabla\left((-\Delta)^{-1} v\right)\right\rangle_{L^{2}(\Omega)} \\
& =\int_{\Omega} \nabla\left((-\Delta)^{-1} u\right) \cdot \nabla\left((-\Delta)^{-1} v\right) d x \\
& =-\int_{\Omega}\left((-\Delta)^{-1} u\right) \cdot \Delta\left((-\Delta)^{-1} v\right) d x+\int_{\partial \Omega}\left((-\Delta)^{-1} u\right) \frac{\partial\left((-\Delta)^{-1} v\right)}{\partial \nu} d S \\
& =\left\langle(-\Delta)^{-1} u, v\right\rangle_{L^{2}(\Omega)}
\end{aligned}
$$

under the suitable boundary conditions (either $(-\Delta)^{-1} u=0$ or $\frac{\partial\left((-\Delta)^{-1} v\right)}{\partial \nu}=0$ on $\partial \Omega$ ). Next, we see that (for each $s \in(0,1)$ )

$$
\begin{aligned}
\langle u, v\rangle_{H^{s}\left(\mathbb{R}^{n}\right)} & =\langle u, v\rangle_{L^{2}\left(\mathbb{R}^{n}\right)}+\left\langle(-\Delta)^{s / 2} u,(-\Delta)^{s / 2} v\right\rangle_{L^{2}\left(\mathbb{R}^{n}\right)} \\
& =\langle u, v\rangle_{L^{2}\left(\mathbb{R}^{n}\right)}+\left\langle(-\Delta)^{s} u, v\right\rangle_{L^{2}\left(\mathbb{R}^{n}\right)}=\left\langle\left(1+(-\Delta)^{s}\right) u, v\right\rangle_{L^{2}\left(\mathbb{R}^{n}\right)} .
\end{aligned}
$$

Using (2.1)-2.4), we obtain

$$
\begin{aligned}
& \nabla_{L^{2}(\Omega)} E_{\epsilon}(u)=(1+(-\Delta))\left(\nabla_{H^{1}(\Omega)} E_{\epsilon}(u)\right), \\
& \nabla_{L^{2}(\Omega)} E_{\epsilon}(u)=\left((-\Delta)^{-1}\right)\left(\nabla_{H^{-1}(\Omega)} E_{\epsilon}(u)\right), \\
& \nabla_{L^{2}(\Omega)} E_{\epsilon}(u)=\left(1+(-\Delta)^{s}\right)\left(\nabla_{H^{s}(\Omega)} E_{\epsilon}(u)\right) .
\end{aligned}
$$


Therefore, we have

$$
\begin{aligned}
\nabla_{H^{1}(\Omega)} E_{\epsilon}(u) & =\left(1+(-\Delta)^{1}\right)^{-1}\left(\nabla_{L^{2}(\Omega)} E_{\epsilon}(u)\right), \\
\nabla_{H^{-1}(\Omega)} E_{\epsilon}(u) & =(-\Delta)\left(\nabla_{L^{2}(\Omega)} E_{\epsilon}(u)\right), \\
\nabla_{H^{s}(\Omega)} E_{\epsilon}(u) & =\left(1+(-\Delta)^{s}\right)^{-1}\left(\nabla_{L^{2}(\Omega)} E_{\epsilon}(u)\right),
\end{aligned}
$$

where $H^{s}(\Omega) \equiv\left\{u \in H^{s}\left(\mathbb{R}^{n}\right) \mid u \equiv 0\right.$ on $\left.\mathbb{R}^{n} \backslash \bar{\Omega}\right\}$. Then the gradient of $E_{\epsilon}$ with respect to $L^{2}(\Omega), H^{-1}(\Omega), H^{1}(\Omega)$, and $H^{s}(\Omega)$ are respectively

$$
\begin{aligned}
\nabla_{L^{2}(\Omega)} E_{\epsilon}(u) & =-\epsilon \Delta u+\frac{f(u)}{\epsilon} \\
\nabla_{H^{-1}(\Omega)} E_{\epsilon}(u) & =(-\Delta)\left(\nabla_{L^{2}(\Omega)} E_{\epsilon}(u)\right)=L\left(\nabla_{L^{2}(\Omega)} E_{\epsilon}(u)\right) \\
\nabla_{H^{1}(\Omega)} E_{\epsilon}(u) & =\left(1+(-\Delta)^{1}\right)^{-1}\left(\nabla_{L^{2}(\Omega)} E_{\epsilon}(u)\right)=L_{1}\left(\nabla_{L^{2}(\Omega)} E_{\epsilon}(u)\right), \\
\nabla_{H^{s}(\Omega)} E_{\epsilon}(u) & =\left(1+(-\Delta)^{s}\right)^{-1}\left(\nabla_{L^{2}(\Omega)} E_{\epsilon}(u)\right)=L_{s}\left(\nabla_{L^{2}(\Omega)} E_{\epsilon}(u)\right),
\end{aligned}
$$

where $L u=-\Delta u$ is an unbounded linear operator, $L_{s} u=\left(1+(-\Delta)^{s}\right)^{-1}$ is a bounded symmetric positive definite linear operator for each $s \in(0,1]$.

$\partial_{t} u=-\nabla_{L^{2}(\Omega)} E_{\epsilon}(u)$ is the Allen-Cahn equation,

$\partial_{t} u=-\nabla_{H^{-1}(\Omega)} E_{\epsilon}(u)$ is the Cahn-Hilliard equation,

$\partial_{t} u=-\nabla_{H^{1}(\Omega)} E_{\epsilon}(u)$ is the Cahn-Hilliard/Allen-Cahn equation, and

$\partial_{t} u=-\nabla_{H^{s}(\Omega)} E_{\epsilon}(u)$ is called the Fractional Cahn-Hilliard/Allen-Cahn equation.

Our research is motivated by the above formulas and its fundamental properties. The following lemmas (Lemmas 2.1 2.7) are useful in the study of our main results.

Lemma 2.1. Let $X$ be a real Hilbert space with inner product $\langle\cdot, \cdot\rangle_{X}$ and corresponding norm $\|\cdot\|_{X}$. For any symmetric positive definite linear operator $L: X \mapsto X$, define a function $\langle\cdot, \cdot\rangle_{L}: X \times X \mapsto \mathbb{R}$ by

$$
\langle u, v\rangle_{L}=\langle L u, v\rangle_{X}
$$

Then $\langle\cdot, \cdot\rangle_{L}$ is also an inner product on $X$.

Proof. Recalling that $L$ is symmetric if

$$
\langle L u, v\rangle_{X}=\langle u, L v\rangle_{X} \quad \text { for all } u, v \in X,
$$

and $L$ is positive definite if there exists positive constant $M$ such that

$$
\langle L u, u\rangle_{X} \geq M\|u\|_{X}^{2} \quad \text { for all } u \in X .
$$

Following 2.6 and (2.7), $\langle\cdot, \cdot\rangle_{L}$ is an inner product on $X$. 
Lemma 2.2. 6, Proposition 2] If $E \in C^{1}(X, \mathbb{R})$ and if $L$ is an invertible, symmetric, positive definite, and linear operator on $X$, then the gradient of $E$ relative to the inner product $\langle\cdot, \cdot\rangle_{L}, \nabla_{L} E$ exists and we have

$$
\nabla_{L} E(u)=L^{-1}\left(\nabla_{X} E(u)\right) \quad \text { for all } u \in X \text {. }
$$

Proof. We note that the first variation of $E$ at $u$ in the direction $v, \delta E(u, v)$, can be expressed as

$$
\left.\frac{d}{d t}(E(u+t v))\right|_{t=0}=\delta E(u, v)=D E(u)(v)=\left\langle\nabla_{X} E(u), v\right\rangle_{X}=\left\langle\nabla_{L} E(u), v\right\rangle_{L},
$$

where $D E(u)$ is the Fréchet derivative of $E$ at $u$. Owing to

$$
\left\langle\nabla_{X} E(u), v\right\rangle_{X}=\left\langle L L^{-1}\left(\nabla_{X} E(u)\right), v\right\rangle_{X}=\left\langle L^{-1}\left(\nabla_{X} E(u)\right), v\right\rangle_{L},
$$

by the definition of $\langle\cdot, \cdot\rangle_{L}$. Moreover, by $(2.8)$ and $(2.9)$ we have

$$
\left\langle\nabla_{L} E(u)-L^{-1}\left(\nabla_{X} E(u)\right), v\right\rangle_{L}=0 \quad \text { for all } v \in X \text {. }
$$

Hence, choosing $v=\nabla_{L} E(u)-L^{-1}\left(\nabla_{X} E(u)\right)$, we find

$$
\left\|\nabla_{L} E(u)-L^{-1}\left(\nabla_{X} E(u)\right)\right\|_{L}^{2}=0,
$$

the lemma is proved.

Lemma 2.3. Let $X$ be a real Hilbert space with inner product $\langle\cdot, \cdot\rangle_{X}$ and corresponding norm $\|\cdot\|_{X}$, and let $L: X \mapsto X$ be a bounded symmetric positive definite linear operator. Let $\langle\cdot, \cdot\rangle_{L}$ be an inner product on $X$ defined by $(2.5)$. Then $\left(X,\langle\cdot, \cdot\rangle_{L}\right)$ is a real Hilbert space with the corresponding norm $\|\cdot\|_{L}$. Moreover, $\|\cdot\|_{L}$ and $\|\cdot\|_{X}$ are equivalent norms.

Proof. By assumption of boundedness of $L$, there exists a constant $K>0$ such that

$$
\|L u\|_{X} \leq K\|u\|_{X} \quad \text { for all } u \text { in } X .
$$

Now, using the Cauchy-Schwarz inequality, 2.7), and (2.10), we have

$$
M\|u\|_{X}^{2} \leq\langle L u, u\rangle_{X} \leq\left|\langle L u, u\rangle_{X}\right| \leq\|L u\|_{X}\|u\|_{X} \leq K\|u\|_{X}^{2}
$$

for all $u$ in $X$. Note that by 2.5 , we have

$$
\|u\|_{L}^{2}=\langle u, u\rangle_{L}=\langle L u, u\rangle_{X}
$$

It follows from (2.11) and 2.12 that we have $\|\cdot\|_{L}$ and $\|\cdot\|_{X}$ are equivalent norms. Thus, if $\left\{u_{n}\right\}_{n=1}^{\infty}$ is a Cauchy sequence in $\left(X,\|\cdot\|_{L}\right)$, then it is a Cauchy sequence in $\left(X,\|\cdot\|_{X}\right)$ and there exists $u \in X$ such that

$$
\lim _{n \rightarrow \infty}\left\|u_{n}-u\right\|_{X}=0
$$


By the equivalence of $\|\cdot\|_{X}$ and $\|\cdot\|_{L}$, we immediately have

$$
\lim _{n \rightarrow \infty}\left\|u_{n}-u\right\|_{L}=0
$$

Hence, $\left(X,\|\cdot\|_{L}\right)$ is a complete normed space.

Lemma 2.4. Suppose that $L: X \mapsto X$ is a symmetric positive definite invertible linear operator. Assume that $L^{-1}: X \mapsto X$ is positive definite. Let $E \in C^{1}(X, \mathbb{R})$, and let $u:[0, T] \mapsto X$ be any solution of the generalized gradient flow 1.2 on $[0, T]$. Then we have

$$
\left\|\partial_{t} u(t)\right\|_{L^{-1}}=\left\|\nabla_{X} E(u(t))\right\|_{L} \quad \text { for all } t \in[0, T]
$$

and

$$
\frac{d}{d t}(E(u(t)))=-\left\|\partial_{t} u(t)\right\|_{L^{-1}}^{2}=-\left\|\nabla_{X} E(u(t))\right\|_{L}^{2} \quad \text { for all } t \in[0, T]
$$

Proof. Indeed:

$$
\begin{aligned}
\left\|\partial_{t} u(t)\right\|_{L^{-1}}^{2} & =\left\langle\partial_{t} u(t), \partial_{t} u(t)\right\rangle_{L^{-1}} \\
& \left.=\left\langle L\left(\nabla_{X} E(u)\right), L\left(\nabla_{X} E(u)\right)\right\rangle_{L^{-1}} \quad \text { (By } 11.2\right) \\
& \left.=\left\langle L^{-1} L\left(\nabla_{X} E(u)\right), L\left(\nabla_{X} E(u)\right)\right\rangle_{X} \quad \text { (By 2.5) }\right) \\
& =\left\langle\nabla_{X} E(u), L\left(\nabla_{X} E(u)\right)\right\rangle_{X} \\
& =\left\langle L\left(\nabla_{X} E(u)\right), \nabla_{X} E(u)\right\rangle_{X} \\
& \left.=\left\langle\nabla_{X} E(u), \nabla_{X} E(u)\right\rangle_{L} \quad \text { (By 2.5) }\right) \\
& =\left\|\nabla_{X} E(u)\right\|_{L}^{2},
\end{aligned}
$$

and

$$
\begin{aligned}
\frac{d}{d t}(E(u(t))) & =\left\langle\nabla_{X} E(u(t)), \partial_{t} u(t)\right\rangle_{X} \\
& \left.\left.=\left\langle\nabla_{X} E(u(t)),-L\left(\nabla_{X} E(u(t))\right)\right\rangle_{X} \quad \text { (By } 1.2\right)\right) \\
& =-\left\langle L\left(\nabla_{X} E(u(t))\right), \nabla_{X} E(u(t))\right\rangle_{X} \\
& =-\left\langle\nabla_{X} E(u(t)), \nabla_{X} E(u(t))\right\rangle_{L} \quad \text { (By (2.5)) } \\
& =-\left\|\nabla_{X} E(u(t))\right\|_{L}^{2} \\
& =-\left\|\partial_{t} u(t)\right\|_{L^{-1}}^{2} \quad(\text { By 2.13) })
\end{aligned}
$$

Lemma 2.5. Let $L: X \mapsto X$ be a symmetric positive definite invertible linear operator on a real Hilbert space $X$. Assume that $L^{-1}: X \mapsto X$ is positive definite. Then we have

$$
\begin{gathered}
\|v\|_{L}=\|L v\|_{L^{-1}} \quad \text { for all } v \text { in } X \\
\left|\langle u, v\rangle_{L}\right| \leq\|u\|_{L} \cdot\|v\|_{L}=\|u\|_{L} \cdot\|L v\|_{L^{-1}} \quad \text { for all } u, v \text { in } X
\end{gathered}
$$


and

$$
\|L u\|_{X}^{2}=\langle L u, u\rangle_{L} \quad \text { and } \quad\|u\|_{X}^{2}=\langle L u, u\rangle_{L^{-1}} \quad \text { for all } u \in X .
$$

Proof. We have indeed, for any $v \in X$,

$$
\begin{aligned}
\|v\|_{L}^{2}=\langle v, v\rangle_{L} & =\langle L v, v\rangle_{X} \quad(\operatorname{By}(2.5)) \\
& =\langle v, L v\rangle_{X} \\
& =\left\langle L^{-1} L v, L v\right\rangle_{X} \\
& =\langle L v, L v\rangle_{L^{-1}} \quad(\mathrm{By}(2.5)) \\
& =\|L v\|_{L^{-1}}^{2} .
\end{aligned}
$$

Therefore we have (2.14).

Since $\left(X,\langle\cdot, \cdot\rangle_{L}\right)$ is an inner product space, the latter result 2.15 can be proved by using the Cauchy-Schwarz inequality and (2.14). Finally we observe that

$$
\begin{aligned}
\|L u\|_{X}^{2} & =\langle L u, L u\rangle_{X}=\langle u, L u\rangle_{L}=\langle L u, u\rangle_{L}, \\
\|u\|_{X}^{2} & =\langle u, u\rangle_{X}=\left\langle L^{-1} L u, u\right\rangle_{X}=\langle L u, u\rangle_{L^{-1}}
\end{aligned}
$$

Lemma 2.6 (Young's inequality). Let $p>1$ and $q$ satisfy

$$
\frac{1}{p}+\frac{1}{q}=1
$$

Let $a \geq 0$ and $b \geq 0$. We have

(i) $a \cdot b \leq \frac{a^{p}}{p}+\frac{b^{q}}{q}$,

(ii) $a \cdot b=\frac{a^{p}}{p}+\frac{b^{q}}{q}$ if and only if $a^{p}=b^{q}$.

Lemma 2.7 (Gronwall's lemma). Let $\varphi \in L^{1}([0, T])$ be a nonnegative function which satisfies for a.e. $t \in[0, T]$,

$$
\varphi(t) \leq c \cdot \int_{0}^{t} \varphi(\tau) d \tau
$$

for some constants $c \geq 0$. Then

$$
\varphi(t)=0 \quad \text { a.e. on }[0, T] \text {. }
$$

\section{Main results}

After being studied for a long long time in harmonic analysis and Laplacian operators, fractional operators defined via singular integrals are riveting attention due to the pliant use that can be made of their nonlocal nature and their applications to models of concrete interest. In particular, equations involving the fractional Laplacian or similar nonlocal 
operators naturally surface in many applications. See $3,4,7,12,15,19,21,22$ for the details. To the best of our knowledge, the following two main theorems (Theorems 3.1 and 3.3) are new, not appear to anywhere.

Theorem 3.1. Let $X_{\epsilon}$ and $X$ be real Hilbert spaces. Suppose that $E_{\epsilon} \in C^{1}\left(X_{\epsilon}, \mathbb{R}\right)$ and $E \in C^{1}(X, \mathbb{R})$. Assume that $L_{\epsilon}: X_{\epsilon} \mapsto X_{\epsilon}, L_{\epsilon}^{-1}: X_{\epsilon} \mapsto X_{\epsilon}, L: X \mapsto X$, and $L^{-1}: X \mapsto X$ are bounded symmetric positive definite linear operators. Assume that

(i) $E_{\epsilon}$ and E satisfy a $\Gamma$-liminf relation:

$$
\text { If } u_{\epsilon} \stackrel{S}{\rightarrow} u \text {, then } \liminf _{\epsilon \rightarrow 0} E_{\epsilon}\left(u_{\epsilon}\right) \geq E(u)
$$

(ii) If $u_{\epsilon}(t) \stackrel{S}{\rightarrow} u(t)$ for all $t \in[0, T]$, then either

$$
\liminf _{\epsilon \rightarrow 0}\left\|\partial_{t} u_{\epsilon}(s)\right\|_{L_{\epsilon}^{-1}}^{2} \geq\left\|\partial_{t} u(s)\right\|_{L^{-1}}^{2}-C \cdot D(s), \quad \forall t \in[0, T)
$$

or

$$
\liminf _{\epsilon \rightarrow 0} \int_{0}^{t}\left\|\partial_{t} u_{\epsilon}(s)\right\|_{L_{\epsilon}^{-1}}^{2} d s \geq \int_{0}^{t}\left(\left\|\partial_{t} u(s)\right\|_{L^{-1}}^{2}-C \cdot D(s)\right) d s, \quad \forall t \in[0, T) .
$$

(iii) If $u_{\epsilon}(t) \stackrel{S}{\rightarrow} u(t)$, then $\liminf _{\epsilon \rightarrow 0}\left\|\nabla_{X_{\epsilon}} E_{\epsilon}\left(u_{\epsilon}(t)\right)\right\|_{L_{\epsilon}}^{2} \geq\left\|\nabla_{X} E(u(t))\right\|_{L}^{2}-\widetilde{C} D(t)$ for all $t \in[0, T)$, where $C>0, \widetilde{C}>0, D$ and $D_{\epsilon}$ are defined as

$$
\begin{aligned}
D(t) & :=\limsup _{\epsilon \rightarrow 0} D_{\epsilon}(t), \quad \forall t \in[0, T), \\
D_{\epsilon}(t) & :=E_{\epsilon}\left(u_{\epsilon}(t)\right)-E(u(t)), \quad \forall t \in[0, T) .
\end{aligned}
$$

(iv) $D(0)=0\left(\right.$ or $\left.\lim _{\epsilon \rightarrow 0} E_{\epsilon}\left(u_{\epsilon}(0)\right)=E(u(0))\right)$.

Let $u_{\epsilon}$ be a solution of the generalized gradient flow

$$
\partial_{t} u_{\epsilon}=-L_{\epsilon}\left(\nabla_{X_{\epsilon}} E_{\epsilon}\left(u_{\epsilon}\right)\right)
$$

on $[0, T)$ with

$$
u_{\epsilon}(t) \stackrel{S}{\rightarrow} u(t) \quad \text { for all } t \in[0, T)
$$

such that for each $t \in[0, T)$,

$$
E_{\epsilon}\left(u_{\epsilon}(0)\right)-E_{\epsilon}\left(u_{\epsilon}(t)\right)=-\int_{0}^{t} \frac{d}{d t}\left(E_{\epsilon}\left(u_{\epsilon}(\tau)\right)\right) d \tau=\int_{0}^{t}\left\|\partial_{t} u_{\epsilon}(\tau)\right\|_{L_{\epsilon}^{-1}}^{2} d \tau .
$$


Then we have

$$
u \in H^{1}([0, T), X), \quad \text { (in particular, continuous in time) }
$$

$u$ is a solution of the following generalized gradient flow $\partial_{t} u=-L\left(\nabla_{X} E(u)\right)$ on $[0, T)$,

$$
\begin{gathered}
\left.D(t)=0 \quad \text { (i.e., } \lim _{\epsilon \rightarrow 0} E_{\epsilon}\left(u_{\epsilon}(t)\right)=E(u(t))\right) \text { for all } t \in[0, T), \\
\lim _{\epsilon \rightarrow 0} \int_{0}^{t}\left\|\partial_{t} u_{\epsilon}(\tau)\right\|_{L_{\epsilon}^{-1}}^{2} d \tau=\int_{0}^{t}\left\|\partial_{t} u(\tau)\right\|_{L^{-1}}^{2} d \tau, \quad \forall t \in[0, T), \\
\lim _{\epsilon \rightarrow 0} \int_{0}^{t}\left\|\nabla_{X_{\epsilon}} E_{\epsilon}\left(u_{\epsilon}(\tau)\right)\right\|_{L_{\epsilon}}^{2} d \tau=\int_{0}^{t}\left\|\nabla_{X} E(u(\tau))\right\|_{L}^{2} d \tau, \quad \forall t \in[0, T) .
\end{gathered}
$$

Proof. Using (3.2), we have

$$
\left[E_{\epsilon}\left(u_{\epsilon}(0)\right)-E_{\epsilon}\left(u_{\epsilon}(t)\right)\right]=[E(u(0))-E(u(t))]+\left[D_{\epsilon}(0)-D_{\epsilon}(t)\right]
$$

Passing liminf to 3.7, we get

$$
\begin{aligned}
& {[E(u(0))-E(u(t))]+\liminf _{\epsilon \rightarrow 0}\left[D_{\epsilon}(0)-D_{\epsilon}(t)\right] } \\
= & \liminf _{\epsilon \rightarrow 0}\left[E_{\epsilon}\left(u_{\epsilon}(0)\right)-E_{\epsilon}\left(u_{\epsilon}(t)\right)\right] \\
= & \liminf _{\epsilon \rightarrow 0} \int_{0}^{t}\left\|\partial_{t} u_{\epsilon}(\tau)\right\|_{L_{\epsilon}^{-1}}^{2} d \tau \quad(\text { By }(3.4)) \\
= & \liminf _{\epsilon \rightarrow 0} \int_{0}^{t}\left\|\partial_{t} u_{\epsilon}(\tau)\right\|_{L_{\epsilon}^{-1}} \cdot\left\|\nabla_{X_{\epsilon}} E_{\epsilon}\left(u_{\epsilon}(\tau)\right)\right\|_{L_{\epsilon}} d \tau \quad \text { (By Lemma 2.4 and (3.3)) } \\
= & \liminf _{\epsilon \rightarrow 0} \int_{0}^{t}\left(\frac{\left\|\partial_{t} u_{\epsilon}(\tau)\right\|_{L_{\epsilon}^{-1}}^{2}}{2}+\frac{\left\|\nabla_{X_{\epsilon}} E_{\epsilon}\left(u_{\epsilon}(\tau)\right)\right\|_{L_{\epsilon}}^{2}}{2}\right) d \tau \\
\geq & \frac{1}{2}\left[\liminf _{\epsilon \rightarrow 0} \int_{0}^{t}\left\|\partial_{t} u_{\epsilon}(\tau)\right\|_{L_{\epsilon}^{-1}}^{2} d \tau+\int_{0}^{t}\left(\liminf _{\epsilon \rightarrow 0}\left\|\nabla_{X_{\epsilon}} E_{\epsilon}\left(u_{\epsilon}(\tau)\right)\right\|_{L_{\epsilon}}^{2}\right) d \tau\right]
\end{aligned}
$$

(By Fatou's Lemma)

$$
\geq \int_{0}^{t}\left(\frac{\left\|\partial_{t} u(\tau)\right\|_{L^{-1}}^{2}+\left\|\nabla_{X} E(u(\tau))\right\|_{L}^{2}}{2}\right) d \tau-\int_{0}^{t}\left(\frac{C+\widetilde{C}}{2}\right) D(\tau) d \tau
$$

(By assumptions (ii) and (iii))

$$
\geq \int_{0}^{t}\left\|\partial_{t} u(\tau)\right\|_{L^{-1}} \cdot\left\|\nabla_{X} E(u(\tau))\right\|_{L} d \tau-\int_{0}^{t}\left(\frac{C+\widetilde{C}}{2}\right) D(\tau) d \tau
$$

(By Young's inequality)

$$
\begin{aligned}
& =\int_{0}^{t}\left\|L^{-1}\left(\partial_{t} u(\tau)\right)\right\|_{L} \cdot\left\|\nabla_{X} E(u(\tau))\right\|_{L} d \tau-\int_{0}^{t}\left(\frac{C+\widetilde{C}}{2}\right) D(\tau) d \tau \\
& \geq \int_{0}^{t}\left|\left\langle L^{-1}\left(\partial_{t} u(\tau)\right), \nabla_{X} E(u(\tau))\right\rangle_{L}\right| d \tau-\int_{0}^{t}\left(\frac{C+\widetilde{C}}{2}\right) D(\tau) d \tau
\end{aligned}
$$


(By the Cauchy-Schwarz inequality)

$$
\begin{aligned}
& \geq \int_{0}^{t}-\left\langle L^{-1}\left(\partial_{t} u(\tau)\right), \nabla_{X} E(u(\tau))\right\rangle_{L} d \tau-\int_{0}^{t}\left(\frac{C+\widetilde{C}}{2}\right) D(\tau) d \tau \\
& =-\int_{0}^{t}\left\langle\partial_{t} u(\tau), \nabla_{X} E(u(\tau))\right\rangle_{X} d \tau-\int_{0}^{t}\left(\frac{C+\widetilde{C}}{2}\right) D(\tau) d \tau
\end{aligned}
$$

(By definition of $\langle\cdot, \cdot\rangle_{L}, 2.5$ )

$$
\begin{aligned}
& =-\int_{0}^{t} \frac{d}{d t}(E(u(\tau))) d \tau-\int_{0}^{t}\left(\frac{C+\widetilde{C}}{2}\right) D(\tau) d \tau \\
& =E(u(0))-E(u(t))-\int_{0}^{t}\left(\frac{C+\widetilde{C}}{2}\right) D(\tau) d \tau .
\end{aligned}
$$

Note that by assumption (i), we have

$$
D(t) \geq 0 \text { for all } t \in[0, T) .
$$

Thus, by assumption (iv), we have

$$
\lim _{\epsilon \rightarrow 0} D_{\epsilon}(0)=0 \text {, i.e., } \lim _{\epsilon \rightarrow 0}\left[E_{\epsilon}\left(u_{\epsilon}(0)\right)-E(u(0))\right]=0 \text {. }
$$

Moreover,

$$
\begin{aligned}
\liminf _{\epsilon \rightarrow 0}\left[D_{\epsilon}(0)-D_{\epsilon}(t)\right] & =\lim _{\epsilon \rightarrow 0} D_{\epsilon}(0)+\left[\liminf _{\epsilon \rightarrow 0}\left(-D_{\epsilon}(t)\right)\right] \\
& =0-\limsup _{\epsilon \rightarrow 0} D_{\epsilon}(t)=-D(t) \quad \text { for all } t \in[0, T) .
\end{aligned}
$$

Following (3.8), (3.9), and (3.10), we have

$$
0 \leq D(t) \leq\left(\frac{C+\widetilde{C}}{2}\right) \int_{0}^{t} D(\tau) d \tau, \quad \forall t \in[0, T) .
$$

By (3.11) and Gronwall's lemma, we have

$$
D(t)=0 \quad \text { for all } t \in[0, T) .
$$

From assumption (i) and 3.12 we have

$$
\limsup _{\epsilon \rightarrow 0}\left[E_{\epsilon}\left(u_{\epsilon}(t)\right)-E(u(t))\right]=\liminf _{\epsilon \rightarrow 0}\left[E_{\epsilon}\left(u_{\epsilon}(t)\right)-E(u(t))\right]=0 \quad \text { for all } t \in[0, T),
$$

i.e., $\lim _{\epsilon \rightarrow 0} D_{\epsilon}(t)=0$ for all $t \in[0, T)$, and

$$
\lim _{\epsilon \rightarrow 0} E_{\epsilon}\left(u_{\epsilon}(t)\right)=E(u(t)) \quad \text { for all } t \in[0, T)
$$


Using 3.13 and 3.12 to 3.8 , we observe that

$$
\begin{aligned}
& E(u(0))-E(u(t)) \\
= & -\int_{0}^{t} \frac{d}{d t}(E(u(\tau))) d \tau=\int_{0}^{t} \frac{\left\|\partial_{t} u\right\|_{L^{-1}}^{2}}{2}+\frac{\left\|\nabla_{X} E(u)\right\|_{L}^{2}}{2} d \tau \\
= & \int_{0}^{t}\left\|\partial_{t} u\right\|_{L^{-1}} \cdot\left\|\nabla_{X} E(u)\right\|_{L} d \tau \\
= & \left.\left.\int_{0}^{t}\left\|L^{-1}\left(\partial_{t} u\right)\right\|_{L} \cdot\left\|\nabla_{X} E(u)\right\|_{L} d \tau \quad \text { (By } 2.14\right)\right) \\
= & \int_{0}^{t}\left|\left\langle L^{-1}\left(\partial_{t} u\right), \nabla_{X} E(u)\right\rangle_{L}\right| d \tau=\int_{0}^{t}-\left\langle L^{-1}\left(\partial_{t} u\right), \nabla_{X} E(u)\right\rangle_{L} d \tau
\end{aligned}
$$

for all $t \in[0, T)$.

Applying the Vanishing Theorem to (3.14), we obtain the following:

$$
\begin{aligned}
\left\|\partial_{t} u\right\|_{L^{-1}} \cdot\left\|\nabla_{X} E(u)\right\|_{L} & =\frac{\left\|\partial_{t} u\right\|_{L^{-1}}^{2}}{2}+\frac{\left\|\nabla_{X} E(u)\right\|_{L}^{2}}{2} \quad \text { for a.e. } \tau \text { in }[0, T), \\
\left\|L^{-1}\left(\partial_{t} u\right)\right\|_{L} & =\left\|\partial_{t} u\right\|_{L^{-1}}=\left\|\nabla_{X} E(u)\right\|_{L} \quad \text { for a.e. } \tau \text { in }[0, T), \\
\left|\left\langle L^{-1}\left(\partial_{t} u\right), \nabla_{X} E(u)\right\rangle_{L}\right| & =\left\|L^{-1}\left(\partial_{t} u\right)\right\|_{L} \cdot\left\|\nabla_{X} E(u)\right\|_{L} \quad \text { a.e. in }[0, T), \\
\left|\left\langle L^{-1}\left(\partial_{t} u\right), \nabla_{X} E(u)\right\rangle_{L}\right| & =-\left\langle L^{-1}\left(\partial_{t} u\right), \nabla_{X} E(u)\right\rangle_{L} \quad \text { a.e. in }[0, T) .
\end{aligned}
$$

Using (3.16), there exists a number $\lambda \in \mathbb{R}$ such that

$$
\nabla_{X} E(u(t))=\lambda L^{-1}\left(\partial_{t} u(t)\right) \text { for a.e. } t \text { in }[0, T) .
$$

It follows from 3.15-3.18 we find that

$$
\lambda=-1
$$

By (3.18) and (3.19), we have

$$
\begin{gathered}
\partial_{t} u(t)=-L\left(\nabla_{X} E(u(t))\right) \quad \text { for a.e. } t \text { in }[0, T), \\
\left(L^{-1}\left(\partial_{t} u(t)\right)=-\nabla_{X} E(u(t)) \quad \text { for a.e. } t \text { in }[0, T) .\right)
\end{gathered}
$$

Taking into account (3.4) and Lemma 2.4 we have

$$
E_{\epsilon}\left(u_{\epsilon}(0)\right)-E_{\epsilon}\left(u_{\epsilon}(t)\right)=\int_{0}^{t}\left\|\partial_{t} u_{\epsilon}(\tau)\right\|_{L_{\epsilon}^{-1}}^{2} d \tau=\int_{0}^{t}\left\|\nabla_{X_{\epsilon}} E_{\epsilon}\left(u_{\epsilon}(\tau)\right)\right\|_{L_{\epsilon}}^{2} d \tau
$$

for all $t \in[0, T)$. Using (3.13) and 3.21) we find that for each $t \in[0, T)$,

$$
\begin{aligned}
\lim _{\epsilon \rightarrow 0} \int_{0}^{t}\left\|\partial_{t} u_{\epsilon}(\tau)\right\|_{L_{\epsilon}^{-1}}^{2} d \tau & =\lim _{\epsilon \rightarrow 0} \int_{0}^{t}\left\|\nabla_{X_{\epsilon}} E_{\epsilon}\left(u_{\epsilon}(\tau)\right)\right\|_{L_{\epsilon}}^{2} d \tau \\
& =\lim _{\epsilon \rightarrow 0}\left[E_{\epsilon}\left(u_{\epsilon}(0)\right)-E_{\epsilon}\left(u_{\epsilon}(t)\right)\right]=E(u(0))-E(u(t))
\end{aligned}
$$




$$
\begin{aligned}
& =\int_{0}^{t}-\frac{d}{d t}(E(u(\tau))) d \tau=\int_{0}^{t}-\left\langle\nabla_{X} E(u(\tau)), \partial_{t} u(\tau)\right\rangle_{X} d \tau \\
& =\int_{0}^{t}\left\langle L^{-1}\left(\partial_{t} u(\tau)\right), \partial_{t} u(\tau)\right\rangle_{X} d \tau \quad(\text { By }(3.20)) \\
& \left.\left.=\int_{0}^{t}\left\langle\partial_{t} u(\tau), \partial_{t} u(\tau)\right\rangle_{L^{-1}} d \tau \quad \text { (By } 2.5\right)\right) \\
& =\int_{0}^{t}\left\|\partial_{t} u(\tau)\right\|_{L^{-1}}^{2} d \tau \\
& =\int_{0}^{t}\left\|\nabla_{X} E(u(\tau))\right\|_{L}^{2} d \tau \quad \text { (By Lemma 2.4 }
\end{aligned}
$$

so that (3.5) and (3.6) hold. By (3.22) we have

$$
\int_{0}^{T}\left\|\partial_{t} u(\tau)\right\|_{L^{-1}}^{2} d \tau=E(u(0))-E(u(T))<+\infty .
$$

By Lemma 2.3 we have $\|\cdot\|_{L^{-1}}$ and $\|\cdot\|_{X}$ are equivalent norms. Using (3.23) we have

$$
\partial_{t} u \in L^{2}(0, T ; X) .
$$

Next, by using the Mean Value Theorem [11, Theorem A3.3, p. 255], we have for each $t$ in $[0, T]$,

$$
\|u(t)-u(0)\|_{X} \leq\left(\sup _{\tau \in[0, t]}\left\|\partial_{t} u(\tau)\right\|_{X}\right) \cdot|t| \leq\left(\sup _{\tau \in[0, T]}\left\|\partial_{t} u(\tau)\right\|_{X}\right) \cdot|t| .
$$

Let $M_{T} \equiv \sup _{\tau \in[0, T]}\left\|\partial_{t} u(\tau)\right\|_{X}<+\infty$ (By (3.24) $)$. Here we get

$$
\begin{aligned}
\int_{0}^{T}\|u(t)\|_{X}^{2} d t & \leq \int_{0}^{T}\left(\|u(t)-u(0)\|_{X}+\|u(0)\|_{X}\right)^{2} d t \\
& \leq \int_{0}^{T}\left(M_{T} \cdot|t|+\|u(0)\|_{X}\right)^{2} d t<+\infty
\end{aligned}
$$

so that $u \in L^{2}(0, T ; X)$. Therefore $u \in H^{1}(0, T ; X)$ and the theorem is proved.

Remark 3.2. In particular, if $L_{\epsilon}=I_{\epsilon}$ and $L=I$, then Theorem 3.1 is the original theorem ( $\Gamma$-convergence of gradient flow) initiated by Sandier and Serfaty [18], where $I_{\epsilon}$ and $I$ are identity linear operators on $X_{\epsilon}$ and $X$ respectively.

Next, we can generalize Theorem 3.1 to $(p, q)$-generalized gradient flows (cf. [20]) where $p$ and $q$ are conjugate exponents in $(1,+\infty)$.

Theorem 3.3. Let $X_{\epsilon}$ and $X$ be real Hilbert spaces. Suppose that $E_{\epsilon} \in C^{1}\left(X_{\epsilon}, \mathbb{R}\right)$ and $E \in C^{1}(X, \mathbb{R})$. Let $p$ and $q$ be conjugate exponents in $(1,+\infty)$ (i.e., $p>1$ and $\frac{1}{p}+\frac{1}{q}=1$ ). Assume that $L_{\epsilon}: X_{\epsilon} \mapsto X_{\epsilon}$, the inverse operator $L_{\epsilon}^{-1}: X_{\epsilon} \mapsto X_{\epsilon}, L: X \mapsto X$, and the inverse of $L, L^{-1}: X \mapsto X$ are bounded symmetric positive definite linear operators. Assume that the following conditions hold: 
(i) $E_{\epsilon}$ and E satisfy a $\Gamma$-liminf relation:

$$
\text { If } u_{\epsilon} \stackrel{S}{\rightarrow} u \text {, then } \liminf _{\epsilon \rightarrow 0} E_{\epsilon}\left(u_{\epsilon}\right) \geq E(u) .
$$

(ii) If $u_{\epsilon}(t) \stackrel{S}{\rightarrow} u(t)$ for all $t \in[0, T)$, then either

$$
\liminf _{\epsilon \rightarrow 0}\left\|\partial_{t} u_{\epsilon}(s)\right\|_{L_{\epsilon}^{-1}}^{p} \geq\left\|\partial_{t} u(s)\right\|_{L^{-1}}^{p}-C \cdot D(s), \quad \forall s \in[0, T)
$$

or

$$
\liminf _{\epsilon \rightarrow 0} \int_{0}^{t}\left\|\partial_{t} u_{\epsilon}(s)\right\|_{L_{\epsilon}^{-1}}^{p} d s \geq \int_{0}^{t}\left(\left\|\partial_{t} u(s)\right\|_{L^{-1}}^{p}-C \cdot D(s)\right) d s, \quad \forall t \in[0, T) .
$$

(iii) If $u_{\epsilon}(t) \stackrel{S}{\rightarrow} u(t)$, then

$$
\liminf _{\epsilon \rightarrow 0}\left\|\nabla_{X_{\epsilon}} E_{\epsilon}\left(u_{\epsilon}(t)\right)\right\|_{L_{\epsilon}}^{q} \geq\left\|\nabla_{X} E(u(t))\right\|_{L}^{q}-\widetilde{C} \cdot D(t)
$$

for each $t \in[0, T)$. Here $C>0, \widetilde{C}>0$, and $D(t), D_{\epsilon}(t)$ are defined by (3.1), 3.2 respectively.

(iv) $D(0)=0\left(\right.$ or $\left.\lim _{\epsilon \rightarrow 0} E_{\epsilon}\left(u_{\epsilon}(0)\right)=E(u(0))\right)$.

Let $u_{\epsilon}$ be a solution of the $(p, q)$-generalized gradient flow

$$
\left\|\partial_{t} u_{\epsilon}\right\|_{L_{\epsilon}^{-1}}^{p-2} \cdot \partial_{t} u_{\epsilon}=-L_{\epsilon}\left(\nabla_{X_{\epsilon}} E_{\epsilon}\left(u_{\epsilon}\right)\right)
$$

on $[0, T)$ with $u_{\epsilon}(t) \stackrel{S}{\rightarrow} u(t)$ for all $t \in[0, T)$ such that for each $t \in[0, T)$,

$$
E_{\epsilon}\left(u_{\epsilon}(0)\right)-E_{\epsilon}\left(u_{\epsilon}(t)\right)=-\int_{0}^{t} \frac{d}{d t}\left(E\left(u_{\epsilon}(\tau)\right)\right) d \tau=\int_{0}^{t}\left\|\partial_{t} u_{\epsilon}(\tau)\right\|_{L_{\epsilon}^{-1}}^{p} d \tau .
$$

Then we have

$$
\begin{gathered}
\left.\partial_{t} u \in L^{p}(0, T ; X) \text { and } u \in L^{p}(0, T ; X), \quad \text { (Hence } u \in W^{1, p}(0, T ; X) .\right) \\
u \text { is a solution of the }(p, q) \text {-generalized gradient flow } \\
\left\|\partial_{t} u\right\|_{L^{-1}}^{p-2} \cdot \partial_{t} u=-L\left(\nabla_{X} E(u)\right) \text { on }[0, T), \\
D(t)=0 \quad \text { (so that } \lim _{\epsilon \rightarrow 0} E_{\epsilon}\left(u_{\epsilon}(t)\right)=E(u(t)) \text { for all } t \in[0, T), \\
\lim _{\epsilon \rightarrow 0} \int_{0}^{t}\left\|\partial_{t} u_{\epsilon}(\tau)\right\|_{L_{\epsilon}^{-1}}^{p} d \tau=\int_{0}^{t}\left\|\partial_{t} u(\tau)\right\|_{L^{-1}}^{p} d \tau \quad \text { for all } t \in[0, T),
\end{gathered}
$$

and

$$
\lim _{\epsilon \rightarrow 0} \int_{0}^{t}\left\|\nabla_{X_{\epsilon}} E_{\epsilon}\left(u_{\epsilon}(\tau)\right)\right\|_{L_{\epsilon}}^{q} d \tau=\int_{0}^{t}\left\|\nabla_{X} E(u(\tau))\right\|_{L}^{q} d \tau
$$

for all $t \in[0, T)$. 
Proof. If $u_{\epsilon}$ satisfies 3.25 , then

$$
\begin{aligned}
\left\|\partial_{t} u_{\epsilon}\right\|_{L_{\epsilon}^{-1}}^{p} & =\left\|\partial_{t} u_{\epsilon}\right\|_{L_{\epsilon}^{-1}}^{p-2} \cdot\left\langle\partial_{t} u_{\epsilon}, \partial_{t} u_{\epsilon}\right\rangle_{L_{\epsilon}^{-1}}=\left\|\partial_{t} u_{\epsilon}\right\|_{L_{\epsilon}^{-1}}^{p-2} \cdot\left\langle L_{\epsilon}^{-1}\left(\partial_{t} u_{\epsilon}\right), \partial_{t} u_{\epsilon}\right\rangle_{X} \\
& =\left\langle L_{\epsilon}^{-1}\left(\left\|\partial_{t} u_{\epsilon}\right\|_{L_{\epsilon}^{-1}}^{p-2} \cdot \partial_{t} u_{\epsilon}\right), \partial_{t} u_{\epsilon}\right\rangle_{X} \\
& =-\left\langle\nabla_{X_{\epsilon}} E_{\epsilon}\left(u_{\epsilon}\right), \partial_{t} u_{\epsilon}\right\rangle_{X} \quad(\text { By }(3.25)) \\
& =-\frac{d}{d t}\left(E_{\epsilon}\left(u_{\epsilon}\right)\right) .
\end{aligned}
$$

By (3.25) again,

$$
\left\|\partial_{t} u_{\epsilon}\right\|_{L_{\epsilon}^{-1}}^{p-1}=\|\| \partial_{t} u_{\epsilon}\left\|_{L_{\epsilon}^{-1}}^{p-2} \cdot \partial_{t} u_{\epsilon}\right\|_{L_{\epsilon}^{-1}}=\left\|L_{\epsilon}\left(\nabla_{X_{\epsilon}} E_{\epsilon}\left(u_{\epsilon}\right)\right)\right\|_{L_{\epsilon}^{-1}}
$$

By using the relation $p=(p-1) \cdot q$ and 3.27$)$ we have

$$
\left\|\partial_{t} u_{\epsilon}\right\|_{L_{\epsilon}^{-1}}^{p}=\left\|L_{\epsilon}\left(\nabla_{X_{\epsilon}} E_{\epsilon}\left(u_{\epsilon}\right)\right)\right\|_{L_{\epsilon}^{-1}}^{q} .
$$

By using Young's inequality and (3.28) we have

$$
\left\|\partial_{t} u_{\epsilon}\right\|_{L_{\epsilon}^{-1}} \cdot\left\|L_{\epsilon}\left(\nabla_{X_{\epsilon}} E_{\epsilon}\left(u_{\epsilon}\right)\right)\right\|_{L_{\epsilon}^{-1}}=\frac{\left\|\partial_{t} u_{\epsilon}\right\|_{L_{\epsilon}^{-1}}^{p}}{p}+\frac{\left\|L_{\epsilon}\left(\nabla_{X_{\epsilon}} E_{\epsilon}\left(u_{\epsilon}\right)\right)\right\|_{L_{\epsilon}^{-1}}^{q}}{q} .
$$

By Lemma 2.5 we have

$$
\left\|L_{\epsilon}\left(\nabla_{X_{\epsilon}} E_{\epsilon}\left(u_{\epsilon}\right)\right)\right\|_{L_{\epsilon}^{-1}}^{q}=\left\|\nabla_{X_{\epsilon}} E_{\epsilon}\left(u_{\epsilon}\right)\right\|_{L_{\epsilon}}^{q}
$$

The proof of this theorem can be achieved step by step with the procedure in the proof of Theorem 3.1 together with (3.26), (3.28), 3.29) and (3.30).

Remark 3.4. Theorem 3.1 is a special case of Theorem 3.3 with $p=q=2$.

\section{Acknowledgments}

Both authors are supported by the Ministry of Science and Technology of Taiwan under the grant MOST 110-2115-M-030-001.

\section{References}

[1] L. Ambrosio, N. Gigli and G. Savaré, Gradient Flows in Metric Spaces and in the Space of Probability Measures, Lectures in Mathematics ETH Zürich, Birkhäuser Verlag, Basel, 2005.

[2] A. Braides, $\Gamma$-convergence for Beginners, Oxford Lecture Series in Mathematics and its Applications 22, Oxford University Press, Oxford, 2002. 
[3] L. A. Caffarelli, J.-M. Roquejoffre and Y. Sire, Variational problems for free boundaries for the fractional Laplacian, J. Eur. Math. Soc. (JEMS) 12 (2010), no. 5, 11511179.

[4] L. A. Caffarelli, S. Salsa and L. Silvestre, Regularity estimates for the solution and the free boundary of the obstacle problem for the fractional Laplacian, Invent. Math. 171 (2008), no. 2, 425-461.

[5] M.-S. Chang and B.-C. Lu, The systems of nonlinear gradient flows on metric spaces and its gamma-convergence, Abstr. Appl. Anal. 2012, Art. ID 910406, 18 pp.

[6] G. Charpiat, P. Maurel, J.-P. Pons, R. Keriven and O. Faugeras, Generalized gradients: Priors on minimization flows, Int. J. Comput. Vis. 73 (2007), no. 3, 325-344.

[7] W. Craig, U. Schanz and C. Sulem, The modulational regime of three-dimensional water waves and the Davey-Stewartson system, Ann. Inst. H. Poincaré Anal. Non Linéaire 14 (1997), no. 5, 615-667.

[8] G. Dal Maso, An Introduction to $\Gamma$-convergence, Progress in Nonlinear Differential Equations and Their Applications 8, Birkhäuser Boston, Boston, MA, 1993.

[9] E. Di Nezza, G. Palatucci and E. Valdinoci, Hitchhiker's guide to the fractional Sobolev spaces, Bull. Sci. Math. 136 (2012), no. 5, 521-573.

[10] M. Kass, A. Witkin and D. Terzopoulos, Snakes: Active contour models, Int. J. Comput. Vis. 1 (1988), no. 4, 321-331.

[11] S. Kesavan, Topics in Functional Analysis and Applications, John Wiley \& Sons, New York, 1989.

[12] M. Kurzke, A nonlocal singular perturbation problem with periodic well potential, ESAIM Control Optim. Calc. Var. 12 (2006), no. 1, 52-63.

[13] N. Q. Le, A gamma-convergence approach to the Cahn-Hilliard equation, Calc. Var. Partial Differential Equations 32 (2008), no. 4, 499-522.

[14] _ On the convergence of the Ohta-Kawasaki equation to motion by nonlocal Mullins-Sekerka law, SIAM J. Math. Anal. 42 (2010), no. 4, 1602-1638.

[15] A. Mellet, S. Mischler and C. Mouhot, Fractional diffusion limit for collisional kinetic equations, Arch. Ration. Mech. Anal. 199 (2011), no. 2, 493-525.

[16] F. Otto, The geometry of dissipative evolution equations: The porous medium equation, Comm. Partial Differential Equations 26 (2001), no. 1-2, 101-174. 
[17] X. Ros-Oton and J. Serra, The Pohozaev identity for the fractional Laplacian, Arch. Ration. Mech. Anal. 213 (2014), no. 2, 587-628.

[18] E. Sandier and S. Serfaty, Gamma-convergence of gradient flows with applications to Ginzburg-Landau, Comm. Pure Appl. Math. 57 (2004), no. 2, 1627-1672.

[19] O. Savin and E. Valdinoci, Elliptic PDEs with fibered nonlinearities, J. Geom. Anal. 19 (2009), no. 2, 420-432.

[20] S. Serfaty, Gamma-convergence of gradient flows on Hilbert and metric spaces and applications, Discrete Contin. Dyn. Syst. 31 (2011), no. 4, 1427-1451.

[21] L. Silvestre, Regularity of the obstacle problem for a fractional power of the Laplace operator, Comm. Pure Appl. Math. 60 (2007), no. 1, 67-112.

[22] E. Valdinoci, From the long jump random walk to the fractional Laplacian, Bol. Soc. Esp. Mat. Apl. SeMA 49 (2009), 33-44.

Mao-Sheng Chang and Jian-Tong Liao

Department of Mathematics, Fu Jen Catholic University, 510. Zhongzheng Rd., Xinzhuang Dist., New Taipei City 24205, Taiwan

E-mail addresses: mschang@math.fju.edu.tw, ljt@math.fju.edu.tw 